\title{
Sepsis: a more faster report from blood culture
}

\author{
Raffaella Ledonne, Paolina Cavalcanti, Maria Agnese Filia, Daniela Perugini, Carmela Gaccione, Paola Pingitore, \\ Cristina Giraldi \\ U.O.C. Clinical and Molecular Microbiology and Virology, A.O. Annunziata, Cosenza
}

Key word: Emocoltura, Sepsi, Febbre

Sepsi: una risposta più rapida dall'emocoltura

\section{SUMMARY}

Introduction: Sepsis is a leading cause of morbidity and mortality. Several studies demonstrated that the earliness of the intervention therapy, including antimicrobial treatment active on the specific pathogen, is associated with a reduction of mortality. In order to permit the use of a quicker appropriate treatment in respect to using the conventional method, we evaluated both the accuracy of strain identification and in vitro antimicrobial susceptibilities directly from the haemocultural bottles.

Methods: We examined II 2 samples of positive blood cultures through traditional technique of subculture, identification and susceptibility testing, and a diagnostic alternative method in the following way: 5-6 ml from positive blood cultures (BACTEC 9640) were transferred into a test tube fitted with a sterile gel separator and centrifuged at $3200 \mathrm{rpm}$ for 15 minutes. After the removal of supernatant, the microbial pellet was suspended in a saline buffer to obtain an $0.5 \mathrm{McFarland}$ inoculum; this turbidity was needed to set up the direct identification and the susceptibility testing (VITEK 2).

Results: The correlation between the two procedures has demonstrated a correlation of $98 \%$ for the strains identification; the correlation was of $100 \%$ for MRSA and ESBL recognition.

Conclusions: The results showed that the use of the direct test for both Gram positive and Gram negative bacteria can give a complete report regarding the identification and susceptibility testing within 24 hours from when the sample becomes positive instead of $48 \mathrm{~h}$ required using the conventional method. The procedure has demonstrated a high reliability both in terms of strains identification and of antibioticsusceptibility. We therefore can suggest, in the diagnostic of sepsis, the introduction of direct method in normal laboratory practice.

\section{INTRODUZIONE}

La sepsi è una patologia infettiva grave, associata ad elevati indici di morbosità e mortalità, soprattutto quando il quadro clinico evolve verso lo shock settico. Negli ultimi anni la ricerca clinica ha individuato diversi interventi in grado di ridurre in modo significativo il rischio di morte; i più efficaci sono rappresentati da una diagnosi microbiologica tesa alla rapidità e tempestività di risposta (referti preliminari, diagnosi molecolare, ecc.) e da nuovi protocolli terapeutici. Nella pratica clinica, tuttavia, la sepsi continua a rimanere un'entità difficile da definire, diagnosticare e trattare, anche perché coinvolge molte specialità in diversi ambiti clinici. Per ridurre in modo significativo i decessi attribuibili a tale patologia è importante sperimentare e sviluppare strumenti utili a facilitarne la diagnosi. Numerosi studi hanno dimostrato che la precocità dell'intervento terapeutico mirato è decisiva ai fini prognostici ed è associata ad una concreta riduzione della mortalità (1). Nella diagnostica della sepsi la "variabile tempo" è un valore di assoluta rilevanza in termini di outcome clinico, soprattutto per il raggiungimento della diagnosi eziologica e per la valutazione della sensibilità dell’isolato agli antimicrobici (2). È compito del microbiologo, pertanto, produrre i risultati dall'emocoltura, indagine microbiologica attualmente considerata il gold standard nella diagnostica delle batteriemie, in tempi sempre più rapidi (3).

Obiettivo dello studio è quello di valutare, rispetto alle tecniche tradizionali, l'accuratezza dei risultati di identificazione e di chemiosensibilità in vitro con un metodo in grado di ridurre i tempi di risposta microbiologica.

\section{MATERIALI E METODI}

112 campioni di emocoltura positivi (Bactec 9240 - Becton Dickinson, BD) sono stati esaminati mediante metodo tradizionale (MT) e mediante un metodo diretto, in uso nel nostro laboratorio (MD).

Il MT consiste nel prelevare una piccola quantità di liquido dal flacone di un'emocoltura segnalata come positiva dallo strumento, per l'allestimento dell'esame microscopico di Gram e delle sottocolture su terreni specifici. L'esame microscopico con colorazione di Gram rappresenta uno strumento estremamente utile per un primo orientamento eziologico (bacilli, cocchi, miceti), per l'elaborazione di un referto preliminare e per suggerire l'impostazione di una tempestiva terapia antibiotica. I flaconi di emocoltura per aerobi segnalati positivi sono seminati su piastre di agar Columbia arricchito con il 5\% di sangue di montone (bioMérieux) ed agar cioccolato polivitex ed incubate overnight in termostato a $37^{\circ} \mathrm{C}$ con atmosfera arricchita in $\mathrm{CO}_{2}$ al $5 \%$. Le emocolture per la ricerca di anaerobi risultate positive, sono seminate su due piastre di agar Columbia arricchito con il 5\% di sangue di montone ed incubate in termostato a $37^{\circ} \mathrm{C}$ overnight l'una in atmosfera arricchita in $\mathrm{CO}_{2}$ del $5 \%$ e l'altra in anaerobiosi. Al fine del corretto utilizzo delle card del sistema Vitek 2 (bioMérieux), specifiche per identificazione ed antibiogramma, sono eseguiti l'esame morfologico delle sottocolture, l'esame microscopico ed i test di catalasi ed ossidasi.

Il MD consiste nel prelevare una piccola quantità di liquido dal flacone dell'emocoltura per l'allestimento di indagini microscopiche atte ad evidenziare la presenza di microrganismi Gram positivi o negativi. Dallo stesso flacone si prelevano 5-6 ml per introdurli in una provetta sterile sottovuoto munita di gel separatore; tale provetta, è centrifugata a 3200 rpm per 15 minuti. Dopo l'eliminazione del sovranatante, il pellet microbico, localizzato sulla superficie del gel, è risospeso con una aliquota di soluzione fisiologica utilizzando una pipetta Pasteur sterile. Dalla sospensione così ottenuta, è poi preparato un inoculo standard con torbidità pari a 0.5 Mc Farland per allestire i test di identificazione e antibiogramma (Vitek 2); card specifiche sono scelte in base al risultato dell'esame microscopico.

Per i Gram positivi sono testati i seguenti antibiotici: Amoxicillina/ac. clavulanico; Ampicillina; Ampicillina/sulbactam, Cefaclor, Cefotaxime, Ceftriaxone, Cefuroxime-acetil, Cefuroxime-sodio, Clindamicina, Eritromicina, Imipenem, Penicillina G, Teicoplanina, Tetraciclina, Trimetropim/sulfametossazolo, Vancomicina, Levofloxacina, Gentamicina, Streptomicina, Tigeciclina, Mupirocina, Oxacillina e Cefoxitina.

Per i Gram negativi sono testati i seguenti antibiotici:

Amoxicillina/ac. Clavulanico, Ampicillina, Amikacina,

\section{Corresponding author: Raffaella Ledonne}

Azienda Ospedaliera di Cosenza-PO “Annunziata” - UOC Microbiologia e Virologia

Via F. Migliori I, Cosenza - Tel.: 0984 68I38I - Fax: 0984681322

E-mail: gircri@virgilio.it 
Cefazolina, Cefotaxime, Cefepime, Ceftazidime; Gentamicina, Meropenem, Imipenem, Nitrofurantoina, Norfloxacina, Trimetropim/sulfametossazolo, Tigeciclina, Levofloxacina, Piperacillina, Piperacillina/Tazobactam; è stata inoltre valutata la produzione di ESBL.

\section{RISULTATI}

Da 112 emocolture positive sono stati isolati 46 microrganismi Gram positivi e 66 Gram negativi.

I risultati ottenuti dall'identificazione di tali microorganismi con entrambi i metodi, MD ed MT, hanno evidenziato una concordanza del 100\% per i ceppi Gram negativi (sono stati isolati un totale di 37 Escherichia coli, 13 Klebsiella pneumoniae, 5 Acinetobacter baumannii, 3 Proteus mirabilis, 3 Pseudomonas aeruginosa, 2 Enterobacter cloacae, 1 Providencia stuartii, 1 Morganella morganii ed 1 Serratia marcescens) e dell' $88 \%$ per i ceppi Gram positivi (comprendenti 11 Staphylococcus aureus, 8 Stafilococchi coagulasi negativi (CoNS), 3 Enterococcus faecalis, 3 Enterococcus faecium, 1 Enterococcus gallinarum, 3 Streptococcus pneumoniae e 3 Streptococcus galactiae).

Il $12 \%$ delle discordanze sono state riscontrate nell'identificazione di specie degli Stafilococchi: infatti 14 S. aureus identificati con MT sono stati speciati come Streptococcus intermedius con MD.

I risultati ottenuti dal confronto, tra MT e MD, dei saggi di chemiosensibilità, per singolo ceppo, hanno dimostrato una concordanza del $100 \%$ nelle card di antibiogramma utilizzate per i seguenti microrganismi: E. faecalis, E. gallinarum, $S$. agalactiae, $P$. aeruginosa, $P$. mirabilis, A. baumannii, $P$. stuartii, M. morganii, S. marcescens, E. cloacae.

Le percentuali di discordanza osservate sono state le seguenti: un ceppo di E. faecium su tre ceppi testati, è risultato resistente all'Ampicillina con MT e sensibile con MD; un ceppo di S. aureus/intermedius su 25 testati (4\%), è risultato resistente all'Eritromicina con MT e sensibile con MD; due ceppi di S. aureus/intermedius su 25 (8\%) sono risultati resistenti alla Clindamicina con MT e sensibili con MD; un ceppo di K. pneumoniae su 13 testati (8\%) ha mostrato sensibilità a Tigeciclina e Trimetropim/sulfametossazolo con MT e resistenza con MD; lo stesso ceppo è risultato resistente alla Piperacillina con MT e sensibile con MD; un ceppo di E. coli su 37 testati (3\%) la Piperacillina/tazobactam è risultato resistente con MT e sensibile con MD.

\section{CONCLUSIONI}

L'utilizzo del metodo diretto di identificazione e di antibiogramma in automatizione, ha dimostrato una buona affidabilità sia per i microrganismi Gram positivi che per Gram negativi. Le discordanze ottenute, soprattutto nelle prove di chemiosensibilità, richiedono un ulteriore perfezionamento del MD. Una casistica più ampia, in particolare per i ceppi in cui sono state osservate le discordanze maggiori, risulta necessaria a tale scopo. Relativamente ad alcuni antibiotici, il metodo diretto ha evidenziato sensibilità non riscontrate con il metodo tradizionale; tale risultato, che rappresenta sicuramente un'indicazione sfavorevole per la terapia del malato, può essere migliorato con una più attenta analisi dell'inoculo diretto o con l'utilizzo di altri sistemi.

In conclusione, nella gestione delle emocolture, gli elementi critici del Turnaround Time (TAT) sono costituiti essenzialmente dai tempi di positivizzazione delle emocolture, referto preliminare e referto definitivo. L'applicazione del MD permette, in una realtà laboratoristica organizzata in modo da garantire una continuità di diagnosi anche nei giorni festivi, una riduzione del TAT con conseguente "risparmio" di 24 o 48 ore rispetto al MT e maggiore rapidità nella refertazione con una più rapida e tempestiva diagnosi eziologica.

\section{BIBLIOGRAFIA}

1. Bouza E, Sousa D, Munoz P, et al. Bloodstream infections: a trial of the impact of different methods of reporting positive blood culture results. Clin Infect Dis 2004; 39: 1161-9.

2. De Cueto M, Ceballos E, Martinez LM, Perea EJ, Pascual A. Use of Positive Blood Cultures for Direct Identification and Susceptibility Testing with the Vitek 2 System. JCM 2004; 42 (8): 3734-8.

3. Murray PR. Manual of Clinical Microbiology, 2003. 